\title{
GLOBAL ANALYSIS OF LIMIT CYCLES IN NETWORKS OF OSCILLATORS
}

\author{
Guy-Bart Stan ${ }^{* 1}$ Rodolphe Sepulchre* \\ * Dep. of Electrical Engineering and Computer Science, \\ Université de Liège, B-4000 Liège, Belgium \\ GB.Stan@ulg.ac.be; R.Sepulchre@ulg.ac.be \\ Proceedings of the Sixth IFAC Symposium on Nonlinear \\ Control Systems (NOLCOS 2004)
}

\begin{abstract}
This paper is concerned with the global stability of limit cycle oscillations for a particular class of systems and networks. In previous work, we defined a class of parameter-dependent nonlinear systems exhibiting an almost globally asymptotically stable limit cycle. The results were proven for values of the parameter in the vicinity of a bifurcation value. In the present paper we restrict ourselves to a piecewise linear version of this class of systems and adapt numerical tools recently proposed in the literature to prove global stability of the limit cycle for a fixed value of the parameter above the bifurcation value. Furthermore, we show how the global stability results for one isolated oscillator is useful to prove the existence of a globally synchrone oscillation in particular networks of identical oscillators.
\end{abstract}

Keywords: Global stability, limit cycles, networks of oscillators, global synchronization.

\section{INTRODUCTION}

Nonlinear oscillations are ubiquitous in nature. A complex system made up of coupled oscillatory systems can be considered as a large-scale network of coupled oscillators. In previous publications (Stan and Sepulchre (2003) and Sepulchre and Stan (2004)), we characterized a class of parameter-dependent systems exhibiting almost globally attractive limit cycle oscillations. This class of systems consists in high-dimensional generalizations of the well known Van der Pol and Fitzhugh-Nagumo oscillators. Element of this class are called dissipative oscillators. The results presented in Sepulchre and Stan (2004) were

1 Research Fellow of the Belgian National Fund for Scientific Research proven for values of the parameter in the vicinity of a bifurcation value. Section 2 summarizes these previous results. In Section 3, we define a piecewise linear equivalent class of systems and adapt numerical tools recently presented in the literature (Gonçalvès et al. (2001) and Gonçalvès et al. (2003)) to conclude about global asymptotic stability of the limit cycle for a particular parameter value above the bifurcation threshold. In Section 4, we show how the stability analysis for one dissipative oscillator extends to the stability analysis of a synchrone oscillation in a network of $N$ identical dissipative oscillators linearly coupled through their outputs. Simulations results are provided on a simple example to illustrate the method. 


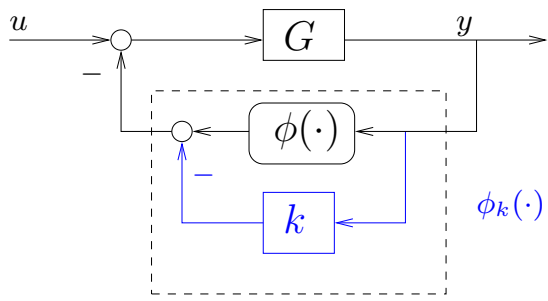

Fig. 1. Block diagram representing the class of SISO nonlinear systems.

\section{BIFURCATIONS IN ABSOLUTELY STABLE FEEDBACK LOOPS}

In Sepulchre and Stan (2004), we consider the feedback system shown in Figure 1 where the SISO passive system $G$ is described by a linear and detectable state space model $\left(A_{G}, B_{G}, C_{G}\right)$ whereas $\phi_{k}(\cdot)$ is the static nonlinearity

$$
\phi_{k}(y)=-k y+\phi(y)
$$

where $\phi(\cdot)$ is a smooth sector nonlinearity in the sector $(0, \infty)$, which satisfies $\phi^{\prime}(0)=\phi^{\prime \prime}(0)=0$, $\phi^{\prime \prime \prime}(0)>0$ and $\lim _{|y| \rightarrow \infty} \frac{\phi(y)}{y}=\infty$ ("stiffening nonlinearity").

The resulting feedback dynamical equations write

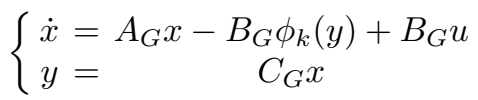

where $x \in \mathbb{R}^{n}, u \in \mathbb{R}$ and $y \in \mathbb{R}$ represents respectively the state, input and output of the feedback system.

We note $G_{k}$ the (positive) feedback interconnection of $G$ with the feedback gain $k$. The feedback system is equally described as the feedback interconnection of $G_{k}$ and the (strictly passive) nonlinearity $\phi(\cdot)$.

We define a dissipative oscillator as a system that admits the feedback representation in Figure 1 and which satisfies two conditions

(1) the feedback system satisfies the dissipation inequality $\dot{S} \leq\left(k-k_{\text {passive }}^{*}\right) y^{2}-y \phi(y)+$ uy where $S(x)$ represents the storage function associated to the feedback system and $k_{\text {passive }}^{*}$ is the critical value of $k$ above which the system $G_{k}$ looses passivity.

(2) when unforced $(u=0)$, the feedback system possesses a global limit cycle, i.e. a stable limit cycle which attracts all solutions except those belonging to the stable manifold of the origin.

The first condition necessarily holds since we assume that the forward block $G$ is passive. Our previous papers (Stan and Sepulchre (2003); Sepulchre and Stan (2004)) provide sufficient conditions for the second condition to be satisfied as well. In particular, denoting $k^{*}$ the bifurcation value at which $G_{k}$ looses stability, we showed that absolute

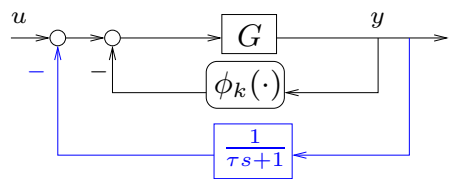

Fig. 2. Adaptation mechanism to convert a bistable system discussed in Figure 1 into a limit cycle.

stability of (2) at $k=k^{*}$ implies (generically) one of the two conditions :

- a supercritical Hopf bifurcation at $k=k^{*}$ and a global limit cycle for $k \gtrsim k^{* 2}$

- a supercritical pitchfork bifurcation at $k=$ $k^{*}$ and a global bistability for $k \gtrsim k^{*}$

The Hopf scenario provides a dissipative oscillator in the vicinity of the bifurcation, i.e. for $k \gtrsim k^{*}$. The pitchfork scenario provides a bistable system in the vicinity of the bifurcation. This bistable system is turned into a relaxation oscillation by using a slow adaptation feedback loop (see Figure 2 ). The resulting system is a dissipative oscillator as well.

A sufficient condition for absolute stability at $k=k^{*}$ is that the system $G_{k}$ looses passivity and stability for the same value of the parameter $k$, i.e. when $k^{*}=k_{\text {passive. Multipliers can be }}^{*}$ used to extend the result to the more general situation where $k^{*}>k_{\text {passive }}^{*}$ (see Sepulchre and Stan (2004)).

\section{A NUMERICAL TOOL FOR GLOBAL ANALYSIS OF LIMIT CYCLES}

\subsection{Problem definition}

For a dissipative oscillator, an almost globally attractive limit cycle appears as the result of the feedback interconnection of a SISO passive system $H$ with the static nonlinearity $\phi_{k}(\cdot)$ defined by (1). In Figure $1, H$ is equal to $G$. In Figure $2, H$ is the feedback interconnection of $G$ with the adapation block $\frac{1}{\tau s+1}$.

$H$ is described by a linear and detectable state space model

$$
(H)\left\{\begin{array}{l}
\dot{x}=A x+B v, \quad x \in \mathbb{R}^{n}, \quad v \in \mathbb{R} \\
y=\quad C x, \quad y \in \mathbb{R}
\end{array}\right.
$$

In this section, we define a qualitatively equivalent piecewise linear system by considering the feedback interconnection of $H$ with a piecewise linear function $f_{p l s}(y)$ :

$v=-f_{p l s}(y)=\left\{\begin{array}{ccc}-p(y+m)-k m & \text { for } \quad y<-m \\ k y & \text { for } & -m \leq y \leq m \\ -p(y-m)+k m & \text { for } & y>m\end{array}\right.$

$2 k \gtrsim k^{*}$ means $k$ "slightly" greater than $k^{*}$, i.e. $k \in$ $\left(k^{*}, k^{*}+\epsilon\right)$ where $\epsilon>0$ is small. 
with $m=\sqrt{\frac{k}{3}}$ and $p>0$.

The resulting piecewise linear system consists of three regions, $\left(R_{1}\right),\left(R_{2}\right)$ and $\left(R_{3}\right)$ in the state space delimited by two switching surfaces, $S_{0}$ and $S_{1}$. The linear dynamics in each region are respectively

$$
\begin{array}{ll}
\left(R_{1}\right) & y(t)<-m \\
& \dot{x}=(A-p B C) x-d B=A_{2} x-d B \\
\left(R_{2}\right) & -m \leq y(t) \leq m \\
& \dot{x}=(A+k B C) x=A_{1} x \\
\left(R_{3}\right) & y(t)>m \\
& \dot{x}=(A-p B C) x+d B=A_{2} x+d B
\end{array}
$$

where $d=m(k+p)$.

Because the feedforward system $H$ is passive and detectable, the matrix $A_{2}$ is Hurwitz for any positive value of $p$. In contrast, the matrix $A_{1}$ has at least 2 eigenvalues with positive real parts for $k>k^{*}$.

Furthermore, we assume that $m>-C A_{2}^{-1} d B$, to guarantee that the unique equilibrium of system $(3),(4)$ is at $x=0$.

From our previous results recalled in Section 2, we expect a limit cycle with a large basin of attraction for a certain range of the parameter $k$. Our analysis, based on Gonçalvès et al. (2003), will be in terms of contraction properties of impact maps that solutions of the PLS define between switching surfaces. The key observation in Gonçalvès et al. (2003) is that these impact maps are linear maps parametrized by the switching time, which is a scalar function of the state.

\subsection{Existence of limit cycles}

We will only be interested in cycles of (3),(4) that are of the type illustrated in Figure $3:$ a (periodic) solution initialized at $Z_{0}^{*} \in S_{0}$ obeys the linear dynamics $\left(R_{2}\right)$ and reaches a point $Z_{1}^{*} \in S_{1}$ after a finite switching time $t_{1}^{*}$; it then obeys the linear dynamics $\left(R_{3}\right)$ and reaches the point $-Z_{0}^{*} \in S_{1}$ after a finite switching time $t_{2}^{*}$.

The values $Z_{0}^{*}, Z_{1}^{*}, t_{1}^{*}$ and $t_{2}^{*}$ that determine the periodic solution satisfy the algebraic equations

$$
\begin{aligned}
& f_{1}\left(t_{1}^{*}, t_{2}^{*}\right)=C Z_{0}^{*}\left(t_{1}^{*}, t_{2}^{*}\right)+m=0 \\
& f_{2}\left(t_{1}^{*}, t_{2}^{*}\right)=C Z_{1}^{*}\left(t_{1}^{*}, t_{2}^{*}\right)-m=0
\end{aligned}
$$

where

$Z_{0}^{*}\left(t_{1}^{*}, t_{2}^{*}\right)=\left(I+e^{A_{2} t_{2}^{*}} e^{A_{1} t_{1}^{*}}\right)^{-1} A_{2}^{-1}\left(I-e^{A_{2} t_{2}^{*}}\right) d B$ $Z_{1}^{*}\left(t_{1}^{*}, t_{2}^{*}\right)=\left(I+e^{A_{1} t_{1}^{*}} e^{A_{2} t_{2}^{*}}\right)^{-1} e^{A_{1} t_{1}^{*}} A_{2}^{-1}\left(I-e^{A_{2} t_{2}^{*}}\right) d B$

The roots of (5),(6) determine periodic solutions of (3),(4). Simulations of the system (3),(4) pro-

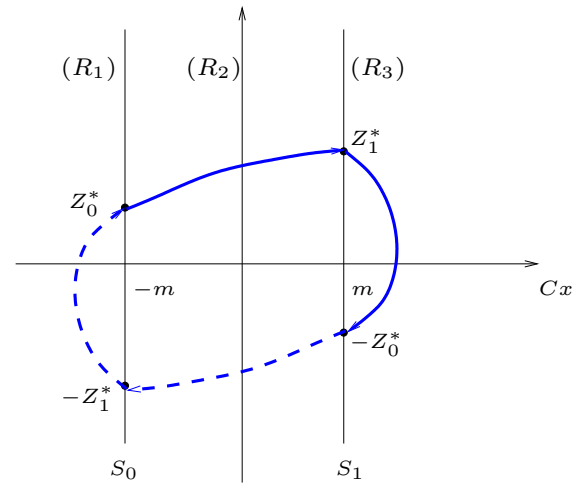

Fig. 3. Limit cycle with four switches per period (first half period in plain line and second half period in dashed line).

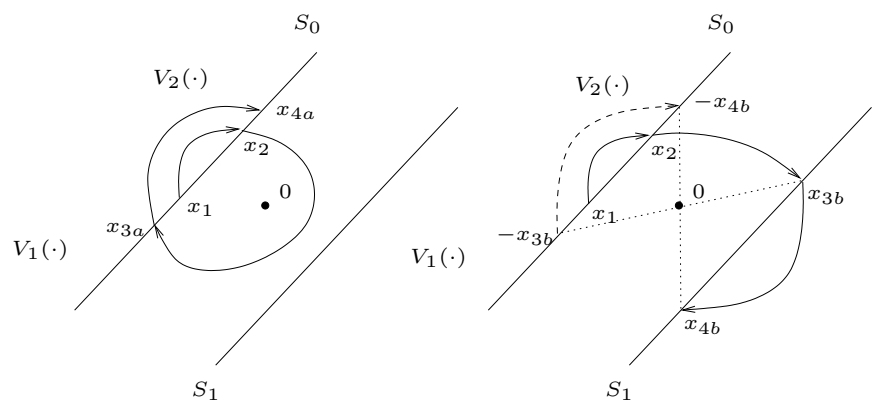

Fig. 4. Impact maps of the PLS; (a) impact map 1 and impact map 2a (b) impact map 1 and impact map $2 \mathrm{~b}$.

vide a good guess for the numerical search of $\left(t_{1}^{*}, t_{2}^{*}\right)$ solving $(5),(6)$.

\subsection{Quadratic stability of impact maps}

Stability of the limit cycle will now be studied through quadratic stability of the impact maps of the system.

Consider a subset $S_{0}^{+}$of $S_{0}$ given by $S_{0}^{+}=\{x \in$ $\left.S_{0}: C A_{1} x \geq 0\right\} . S_{0}^{+}$is the set of points in $S_{0}$ that can be reached by trajectories initialized in $\left(R_{1}\right)$. In a similar way, define $S_{0}^{-} \subset S_{0}$ as $S_{0}^{-}=\left\{x \in S_{0}: C A_{1} x \leq 0\right\}$ and also $S_{1}^{+}=-S_{0}^{-}$ and $S_{1}^{-}=-S_{0}^{+}$. From symmetry considerations, three impact maps only are of interest for the analysis. The first impact map (impact map 1) takes points from $S_{0}^{-}$and maps them in $S_{0}^{+}$. The second impact map (impact map 2a) takes points from $S_{0}^{+} \backslash\left\{Z_{0}^{*}\right\}$ and maps them back to $S_{0}^{-} \backslash\left\{Z_{0}^{*}\right\}$. Finally, the third impact map (impact map 2b) takes points from $S_{0}^{+}$and maps them to $S_{1}^{+}$.

Let $x_{1}$ be a point in $S_{0}^{-} \backslash\left\{-Z_{1}^{*}\right\}$. The trajectory $x_{1}(t)$ will necessarily switch after a finite switching time $t_{1}$ at $x_{2}=x_{1}\left(t_{1}\right)$ since $A_{2}$ is Hurwitz. Since $A_{1}$ is not Hurwitz, a trajectory starting at $x_{2} \in S_{0}^{+} \backslash\left\{Z_{0}^{*}\right\}$ can either switch at some point in $S_{0}$ or at some point in $S_{1}$ (or not switch at all if $x_{2}$ belongs to the stable manifold of the origin). Let $S_{a} \subset S_{0}^{+} \backslash\left\{Z_{0}^{*}\right\}\left(S_{b} \subset S_{0}^{+}\right)$be the set of points 
that switch in $S_{0}\left(S_{1}\right)$. If $x_{2} \in S_{a}\left(x_{2} \in S_{b}\right)$ the trajectory switches in finite time $t_{2 a}\left(t_{2 b}\right)$ at $x_{3 a}=x_{2}\left(t_{2 a}\right) \in S_{0}^{-} \backslash\left\{-Z_{1}^{*}\right\} \quad\left(x_{3 b}=x_{2}\left(t_{2 b}\right) \in S_{1}^{+}\right)$. Then, it switches again at $x_{4 a}=x_{3 a}\left(t_{3 a}\right)\left(x_{4 b}=\right.$ $x_{3 b}\left(t_{3 b}\right)$ ), and so on (see Figure 4$)$.

The symmetry of the system allows to perform the analysis on a half trajectory. This means that it is equivalent to consider the trajectory starting at $x_{2}$ or $-x_{2}$. The idea is to check if $x_{3 a}$ or $-x_{3 b}$ are closer in some sense to $-Z_{1}^{*}$ than $x_{1}$. If so for any point $x_{1} \in S_{0}^{-} \backslash\left\{-Z_{1}^{*}\right\}$, the limit cycle is globally asymptotically stable.

Since $x_{1}, x_{2}, x_{3 a} \in S_{0}$ and $x_{3 b} \in S_{1}$, we can write $x_{1}=-Z_{1}^{*}+\Delta_{1}, x_{2}=Z_{0}^{*}+\Delta_{2}, x_{3 a}=-Z_{1}^{*}+\Delta_{3 a}$ and $x_{3 b}=Z_{1}^{*}+\Delta_{3 b}$, where $C \Delta_{1}=C \Delta_{2}=$ $C \Delta_{3 a}=C \Delta_{3 b}=0$.

A sufficient condition for the first return map to be contracting around $Z_{0}^{*}$ is

$$
\begin{aligned}
V_{2}\left(\Delta_{2}\right) & <V_{1}\left(\Delta_{1}\right) \text { for all } \Delta_{1} \in S_{0}^{-} \backslash\left\{-Z_{1}^{*}\right\} \\
V_{1}\left(\Delta_{3 a}\right) & <V_{2}\left(\Delta_{2}\right) \text { for all } \Delta_{2} \in S_{a} \backslash\left\{Z_{0}^{*}\right\} \\
V_{1}\left(\Delta_{3 b}\right) & <V_{2}\left(\Delta_{2}\right) \text { for all } \Delta_{2} \in S_{b} \backslash\left\{Z_{0}^{*}\right\}
\end{aligned}
$$

where $V_{1}(\cdot)$ and $V_{2}(\cdot)$ are quadratic Lyapunov functions defined on $S_{0}^{-}$and $S_{0}^{+}$respectively (see Figure 4).

The key result proven by Gonçalvès et al. (2003) is that the impact maps induced by an LTI (linear time invariant) flow between two switching surfaces can be represented as a linear transformation analytically parametrized by a scalar function of the state. This parameter is simply the switching time associated with the impact map. Thus, we have $\Delta_{2}=H_{1}\left(t_{1}\right) \Delta_{1}, \Delta_{3 a}=H_{2 a}\left(t_{2 a}\right) \Delta_{2}, \Delta_{3 b}=$ $H_{2 b}\left(t_{2 b}\right) \Delta_{2}$.

We then have to prove that

$$
\begin{aligned}
r_{1}\left(t_{1}\right) \triangleq & \Delta_{1}^{T} P_{1}\left(t_{1}\right) \Delta_{1} \\
& =V_{1}\left(\Delta_{1}\right)-V_{2}\left(H_{1}\left(t_{1}\right) \Delta_{1}\right)>0 \\
r_{2 a}\left(t_{2 a}\right) \triangleq & \Delta_{2}^{T} P_{2 a}\left(t_{2 a}\right) \Delta_{2} \\
& =V_{2}\left(\Delta_{2}\right)-V_{1}\left(H_{2 a}\left(t_{2 a}\right) \Delta_{2}\right)>0 \\
r_{2 b}\left(t_{2 b}\right) \triangleq & \Delta_{2}^{T} P_{2 b}\left(t_{2 b}\right) \Delta_{2} \\
& =V_{2}\left(\Delta_{2}\right)-V_{1}\left(H_{2 b}\left(t_{2 b}\right) \Delta_{2}\right)>0
\end{aligned}
$$

for all expected switching times $t_{1} \in \mathcal{T}_{1}, t_{2 a} \in \mathcal{T}_{2 a}$ and $t_{2 b} \in \mathcal{T}_{2 b}$ where $\mathcal{T}_{1}, \mathcal{T}_{2 a}$ and $\mathcal{T}_{2 b}$ denote the set of all expected switching times corresponding respectively to all $\Delta_{1} \in S_{0}^{-} \backslash\left\{-Z_{1}^{*}\right\}, \Delta_{2} \in S_{a} \backslash\left\{Z_{0}^{*}\right\}$ and $\Delta_{2} \in S_{b} \backslash\left\{Z_{0}^{*}\right\}$.

By discretizing the sets of expected switching times, inequalities (7), (8) and (9) define a finite set of LMIs in the unknowns $P_{i}=P_{i}^{T}>0, i=$ $1,2 a, 2 b$.

\subsection{Bounds on switching times}

A key point in the above quadratic stability analysis is to determine good upper bounds on the switching times. Such bounds can be computed for instance when the linear dynamics in each region are Hurwitz and possess no equilibrium (see Gonçalvès et al. (2003) for details).

The unstable equilibrium $x=0$ of $(3),(4)$ typically possesses a stable manifold when $n>2$. In this case, the switching times are unbounded because of intersections between the stable eigenspace of $A_{1}$ and the switching surfaces $S_{0}$ and $S_{1}$. This situation requires a special treatment that will be presented in a forthcoming publication. For the sake of illustration in the present paper, we restrict our attention to the case where $x=0$ has no stable manifold. This occurs trivially in the 2-dimensional case (even though alternative methods exist to analyse global properties of limit cycles in the plane) but also for conservative systems (3) for which $A_{1}$ is antistable. We generically assume that $A_{1}$ has no real unstable eigenvalue.

If $n=2$, the matrix $A_{1}$ is generically antistable and the upper bound on $t_{1}$ is computed by considering the worst switching scenario for a point belonging to $S_{0}^{+}$, i.e. when $C \dot{x}=0$. There exist only one point on $S_{0}^{+}$corresponding to this worst case situtation. The upper bound is thus the switching time associated with this point.

Moreover, when $A_{1}$ is antistable, any trajectory belonging to $S_{0}^{+}$will necessarily switch at a point belonging to $S_{1}$. As a consequence, there are only two impact maps to consider, i.e., impact map 1 and impact map $2 \mathrm{~b}$.

\subsection{Simulation results for PLS Fitzhugh-Nagumo}

In Sepulchre and Stan (2004), we showed that the system in Figure 2 with $G(s)=\frac{1}{s}$ is a particular case of the Fitzhugh-Nagumo oscillator (Murray (2002)). Analytical results that allow to conclude about global asymptotic stability for $k \gtrsim k^{*}$ for dissipative oscillators based on the feedback structure in Figure 2 were also presented. To illustrate the method, we present here the results obtained for 2 dimensional systems corresponding to Figure 2 where $G(s)=\frac{1}{s+\alpha}, \tau>1$.

The critical bifurcation value for this example is $k^{*}=\alpha+\frac{1}{\tau}$. The chosen parameters values are $\alpha=1$ and $\tau=20$. From these values, we compute $k^{*}=1.05$. From our previous analytical results, we expect a global asymptotic limit cycle for $k \gtrsim 1.05$. Nevertheless, the range of values of $k$ for which this behavior holds is not known. Replacing $\phi_{k}(y)$ by $f_{p l s}(y)$, and choosing values for $p$ and $k$ such that $p>0$ and $k>k^{*}$, we may use the numerical tools presented in Section 3 to 


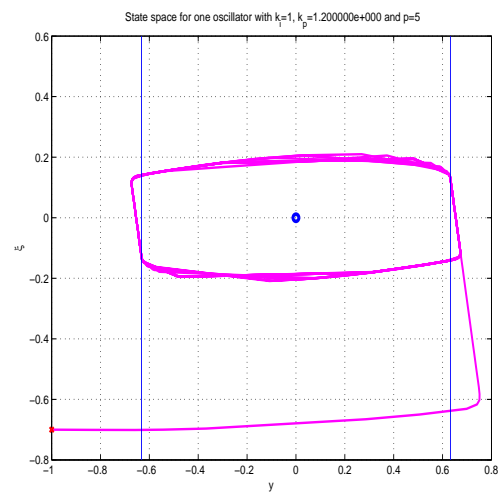

Fig. 5. State space for the piecewise linear Fitzhugh-Nagumo oscillator for $k=1.2, p=$ 5 and $\tau=20$.

conclude about global asymptotic stability of a limit cycle for the chosen value of $k$.

Before presenting the simulation results, we briefly explain the inputs and outputs of the algorithm. The inputs are the transfer function $G(s)$ together with the parameters $\alpha, k>0, p>0$ and $\tau$. A graphic showing the minimum eigenvalues of each $P_{i}\left(t_{i}\right), i=1,2 b$ in (7)-(9) is generated. GAS of the limit cycle is then concluded if the minimum eigenvalues are positive on their respective set of expected switching times.

In this example, we consider the particular values $k=1.2$ and $p=5$. We then compute $m=0.63$ and $d=3.92$. The simulation results of this PLS for a particular initial condition are given on Figure 5.

The numerical algorithm is then applied to this particular PLS. The number of $\left(t_{1}^{*}, t_{2}^{*}\right)$ solutions found by the algorithm for (5) and (6) is equal to one, i.e. $t_{1}^{*}=8.88$ and $t_{2}^{*}=8.4$. These values agree with those found by simulation of the dynamical system. We then solve the LMIs defined by (7) and (9) and plot Figure 6. On this figure, we see that the minimum eigenvalue of each condition (7) and (9) is positive on its respective set of expected switching times $\mathcal{T}_{1}$ and $\mathcal{T}_{2 b}$. The set of expected switching times in this example are approximately $\mathcal{T}_{1}=(0,16.7)$ and $\mathcal{T}_{2 b}=(0,9)$. For instance, if $t_{2 b} \geq 9$, there is no point in $S_{0}^{+}$with switching time equal to $t_{2 b}$. Using conditions (7) and (8) we have showed that this system possesses a globally asymptotically stable limit cycle in $\mathbb{R}^{2} \backslash\{0\}$.

\section{DISSIPATIVITY AND GLOBAL SYNCHRONIZATION}

In Stan and Sepulchre (2004), we show how the stability analysis for one dissipative oscillator of type (3),(1) extends to the stability analysis of a synchrone oscillation in a network of coupled identical dissipative oscillators. In this section, we recall the main result without proof.

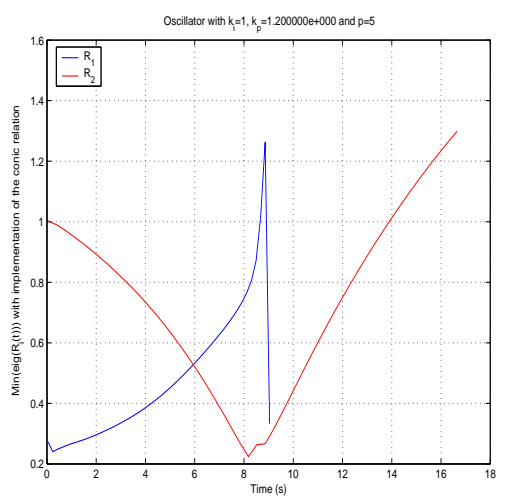

Fig. 6. Temporal evolution of the minimum eigenvalues of $P_{1}(t)$ and $P_{2}(t)$.

We consider a network of $N$ identical oscillators, linearly coupled through their outputs. The dynamics of the network are easily represented with the help of the Kronecker product (Graham $(1981))$, i.e.

$$
\left\{\begin{array}{lc}
\dot{X}=\left(I_{N} \otimes A\right) X+ & \left(I_{N} \otimes B\right)\left(-\Phi_{k}(Y)-\Gamma Y\right) \\
Y= & \left(I_{N} \otimes C\right) X
\end{array}\right.
$$

where $X=\left(x_{1}, \ldots, x_{N}\right)^{T}, U=\left(u_{1}, \ldots, u_{N}\right)^{T}$, $Y=\left(y_{1}, \ldots, y_{N}\right)^{T}$ with $x_{i}, u_{i}, y_{i}$ representing, respectively, the state, input and output of oscillator $i$ in the network. $\Phi_{k}(Y)$ is a column vector whose $i^{t h}$ element is $\phi_{k}\left(y_{i}\right)$ and $\Gamma \in \mathbb{R}^{N \times N}$ is the interconnection matrix of the network. We assume that all rows of $\Gamma$ sum to zero and that the rank of $\Gamma$ is equal to $N-1$. This last assumption is equivalent to the assumption that the network is connected.

The assumptions on $\Gamma$ imply

$$
\bar{R} \Gamma=\left(\begin{array}{cc}
0 & 0^{T} \\
0 & \tilde{\Gamma}
\end{array}\right) \bar{R}
$$

where $\bar{R}=\left(I_{N}-\left(\mathbf{1}, 0_{N \times N-1}\right)\right)$ with $\mathbf{1}=(1, \ldots, 1)^{T}$ is a projection matrix.

The class of interconnection matrices $\Gamma$ is further assumed to be such that $\tilde{\Gamma}$ is positive definite (i.e. $\left.x^{T} \tilde{\Gamma}_{s} x=x^{T} \frac{1}{2}\left(\tilde{\Gamma}+\tilde{\Gamma}^{T}\right) x>0, \forall x \in \mathbb{R}^{n} \backslash\{0\}\right) . \mathrm{We}$ denote by $\lambda_{\min }\left(\tilde{\Gamma}_{s}\right)$ the smallest eigenvalue of the symmetric part of $\tilde{\Gamma}$.

Property (11) with $\tilde{\Gamma}>0$ constitutes the main characteristic of the class of interconnections we consider. Note that our assumptions do not require that $\Gamma$ is symmetric.

In Stan and Sepulchre (2004) we show that, for strong coupling (i.e. $\lambda_{\min }\left(\tilde{\Gamma}_{s}\right)>$ threshold $(k)$ ), all solutions of (10) exponentially converge to the invariant subspace

$$
\left\{X \in \mathbb{R}^{n N}: x_{1}=\cdots=x_{N}\right\}
$$

Because the dynamics of the network decouple in the invariant subspace (12), this, in turn, implies that all oscillators synchronize exponentially and 


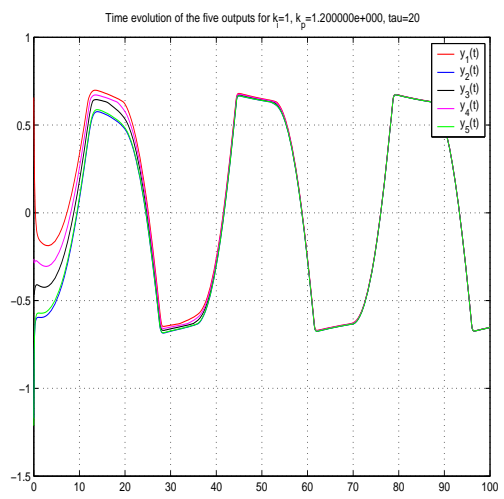

Fig. 7. Time evolution of the outputs in a network of 5 oscillators coupled through $S_{5}$ symmetry.

that all bounded solutions converge to the $\omega$-limit sets of the decoupled system.

The synchronization property (12) derives from an incremental dissipativity property of the network. Our approach is closely related to the contraction approaches of Slotine and Wang (2003) and Pogromsky and Nijmeijer (2001). The incremental dissipativity property together with an observability assumption may also be seen as an incremental stability property of the particular network (see Angeli (2002)).

\subsection{An example}

We consider a network composed of 5 identical PLS Fitzhugh-Nagumo oscillators characterized by $G(s)=\frac{1}{s+1}, k=1.2, p=5$ and $\tau=20$ and coupled in order to form a $S_{5}$ symmetry network where the input of oscillator $i$ is $u_{i}=$ $K \sum_{j=1}^{N}\left(y_{j}-y_{i}\right)$. For this class of interconnection, it can be shown (Stan and Sepulchre (2004)) that $\lambda_{\min }\left(\tilde{\Gamma}_{s}\right)=N K$. The value of $K$ is chosen such that the strong coupling assumption is satisfied. We consider $K=1$.

In Section 3.5 we have numerically proved that for these parameters values the uncoupled PLS Fitzhugh-Nagumo possesses a globally asymptotically stable limit cycle in $\mathbb{R}^{2} \backslash\{0\}$.

A separate argument, not included in the present paper, shows that all solutions of the coupled system are bounded. We thus conclude that for strong coupling, all solutions, except the trivial one, converge towards the $\omega$-limit set of the decoupled system which is a globally attractive limit cycle.

Simulation results are presented in Figure 7. We clearly see that the oscillators synchronize around a common limit cycle oscillation. This limit cycle is identical to the one obtained for an isolated oscillator. Particular to our simulation, we note the relaxation type of the oscillations due to the high value of $\tau(\tau=20)$.

\section{CONCLUSIONS}

In previous publications (Stan and Sepulchre (2003) and Sepulchre and Stan (2004)), we have presented conditions for global limit cycle oscillations in the feedback interconnection of a passive system with a static nonlinearity that has a parameterized negative slope at the origin. These results hold valid for values of the parameter in the vicinity of a critical bifurcation value. In this paper, we adapt numerical algorithms recently proposed in the literature to conclude about global asymptotic stability for a particular value of the parameter above the critical value. Moreover, using recent global synchronization results, we show how the analysis for one system extends to the analysis of a synchrone oscillation in particular networks of identical oscillators.

\section{AKNOWLEDGEMENT}

This paper presents research partially supported by the Belgian Programme on Inter-university Poles of Attraction, initiated by the Belgian State, Prime Minister's Office for Science, Technology and Culture.

\section{REFERENCES}

D. Angeli. A Lyapunov approach to incremental stability properties. IEEE Trans. on Automatic Control, 47:410-422, 2002.

J. Gonçalvès, A. Megretski, and M. Dahleh. Global stability of relay feedback systems. IEEE Trans. on Automatic Control, 46(4):550562, April 2001.

J. Gonçalvès, A. Megretski, and M. Dahleh. Global analysis of piecewise linear systems using impact maps and quadratic surface lyapunov functions. IEEE Trans. on Automatic Control, 48(12):2089-2106, December 2003.

A. Graham. Kronecker Products and Matrix Calculus With Applications. Ellis Horwood Series, 1981.

J. D. Murray. Mathematical Biology, I: An Introduction. Springer Verlag, third edition, 2002.

A. Pogromsky and H. Nijmeijer. Cooperative oscillatory behavior of mutually coupled dynamical systems. IEEE Trans. on circuits and systems, 48:152-162, 2001.

R. Sepulchre and G.-B. Stan. Feedback mechanisms for global oscillations in Lure systems. Submitted for publication in Systems and Control Letters, 2004.

J.-J. Slotine and W. Wang. A study of synchronization and group cooperation using partial contraction theory. In Kumar V., editor, Block Island Workshop on Cooperative Control. Springer-Verlag, 2003.

G.-B. Stan and R. Sepulchre. Dissipativity characterization of a class of oscillators and networks 
of oscillators. In $42^{\text {nd }}$ IEEE Conf. on Decision and Control, pages 4169-4173. Maui, Hawaii, USA, 2003.

G.-B. Stan and R. Sepulchre. Dissipativity and global analysis of limit cycles in networks of oscillators. To appear in proceedings of the Seixteenth International Symposium on Mathematical Theory of Networks and Systems, 2004. 